\title{
ON THE SUM OF THE INDEX OF A PARABOLIC SUBALGEBRA AND OF ITS NILPOTENT RADICAL
}

\author{
RUPERT W. T. YU
}

(Communicated by Dan M. Barbasch)

\begin{abstract}
In this short note, we investigate the following question of Panyushev stated in 2003: "Is the sum of the index of a parabolic subalgebra of a semisimple Lie algebra $\mathfrak{g}$ and the index of its nilpotent radical always greater than or equal to the rank of $\mathfrak{g}$ ?" Using the formula for the index of parabolic subalgebras conjectured by Tauvel and the author and proved by FauquantMillet and Joseph in 2005 and Joseph in 2006, we give a positive answer to this question. Moreover, we also obtain a necessary and sufficient condition for this sum to be equal to the rank of $\mathfrak{g}$. This provides new examples of direct sum decomposition of a semisimple Lie algebra verifying the "index additivity condition" as stated by Raïs.
\end{abstract}

\section{INTRODUCTION}

Let $\mathfrak{g}$ be a Lie algebra over an algebraically closed field $\mathbb{k}$ of characteristic zero. For $f \in \mathfrak{g}^{*}$, we denote by $\mathfrak{g}^{f}=\{X \in \mathfrak{g} ; f([X, Y])=0$ for all $Y \in \mathfrak{g}\}$, the annihilator of $f$ for the coadjoint representation of $\mathfrak{g}$. The index of $\mathfrak{g}$, denoted by $\chi(\mathfrak{g})$, is defined to be

$$
\chi(\mathfrak{g})=\min _{f \in \mathfrak{g}^{*}} \operatorname{dim} \mathfrak{g}^{f} .
$$

It is well known that if $\mathfrak{g}$ is an algebraic Lie algebra and $G$ denotes its algebraic adjoint group, then $\chi(\mathfrak{g})$ is the transcendence degree of the field of $G$-invariant rational functions on $\mathfrak{g}^{*}$.

The index of a semisimple Lie algebra $\mathfrak{g}$ is equal to the rank of $\mathfrak{g}$. This can be obtained easily from the isomorphism between $\mathfrak{g}$ and $\mathfrak{g}^{*}$ via the Killing form. There has been quite a lot of recent work on the determination of the index of certain subalgebras of a semisimple Lie algebra: parabolic subalgebras and related subalgebras ([2], [9], [13], [8]), centralizers of elements and related subalgebras ([10], $[1],[15],[7])$.

Let $\mathfrak{g}$ be a semisimple Lie algebra, $\mathfrak{p}$ a parabolic subalgebra of $\mathfrak{g}$ and $\mathfrak{u}$ (resp. $\mathfrak{l}$ ) the nilpotent radical (resp. a Levi factor) of $\mathfrak{p}$. In [10, Corollary 1.5 (i)], Panyushev showed that

$$
\chi(\mathfrak{p})+\chi(\mathfrak{u}) \leq \operatorname{dim} \mathfrak{l} .
$$

Received by the editors August 18, 2006.

2000 Mathematics Subject Classification. Primary 17B20.

(C)2008 American Mathematical Society Reverts to public domain 28 years from publication 
He then suggested [10, Remark (ii) of Section 6] that

$$
\chi(\mathfrak{p})+\chi(\mathfrak{u}) \geq \operatorname{rkg} .
$$

For example, it is well known that if $\mathfrak{b}$ is a Borel subalgebra of $\mathfrak{g}$ and $\mathfrak{n}$ is its nilpotent radical, then $\chi(\mathfrak{b})+\chi(\mathfrak{n})=\operatorname{rkg}($ see for example [12], [14, Chapter 40]). It is therefore also interesting to characterise parabolic subalgebras where equality holds in (2). Indeed, in [11], Raïs looked for examples of direct sum decompositions $\mathfrak{g}=\mathfrak{m} \oplus \mathfrak{n}$ verifying the "index additivity condition", namely $\mathfrak{m}$ and $\mathfrak{n}$ are Lie subalgebras of $\mathfrak{g}$ and

$$
\chi(\mathfrak{g})=\chi(\mathfrak{m})+\chi(\mathfrak{n}) .
$$

If $\mathfrak{u}_{-}$denotes the nilpotent radical of the opposite parabolic subalgebra $\mathfrak{p}_{-}$of $\mathfrak{p}$, then $\mathfrak{g}=\mathfrak{p} \oplus \mathfrak{u}_{-}$and the Lie algebras $\mathfrak{u}$ and $\mathfrak{u}_{-}$are isomorphic. Thus parabolic subalgebras such that equality holds in (2) would provide examples of direct sum decompositions verifying the index additivity condition.

Using the formula conjectured in $[13]$ and proved in $[3,6]$ for the index of parabolic subalgebras, we obtain a formula for the sum $\chi(\mathfrak{p})+\chi(\mathfrak{u})$. By a careful analysis of root systems, we prove inequality (2) and give a necessary and sufficient condition for the equality to hold in (2) (see Theorem 2.2).

To describe the index of a parabolic subalgebra and the index of its nilpotent radical, we need to recall Kostant's cascade construction of pairwise strongly orthogonal roots $([4],[5],[14])$.

Let us fix a Cartan subalgebra $\mathfrak{h}$ of $\mathfrak{g}$ and a Borel subalgebra $\mathfrak{b}$ of $\mathfrak{g}$ containing $\mathfrak{h}$. Denote by $R, R^{+}$and $\Pi=\left\{\alpha_{1}, \ldots, \alpha_{\ell}\right\}$ respectively the set of roots, positive roots and simple roots with respect to $\mathfrak{h}$ and $\mathfrak{b}$. For any $\alpha \in R$, let $\mathfrak{g}_{\alpha}$ be the root subspace associated to $\alpha$. Choose $X_{\alpha} \in \mathfrak{g}_{\alpha}$ such that $\alpha\left(\left[X_{\alpha}, X_{-\alpha}\right]\right)=2$. We shall write $\alpha^{\vee}=\left[X_{\alpha}, X_{-\alpha}\right] \in \mathfrak{h}$, and for $\lambda \in \mathfrak{h}^{*},\left\langle\lambda, \alpha^{\vee}\right\rangle=\lambda\left(\alpha^{\vee}\right)$. For $S \subset \Pi$, we denote by $R_{S}=R \cap \mathbb{Z} S, R_{S}^{+}=R_{S} \cap R^{+}$. If $S$ is connected, then we shall denote by $\varepsilon_{S}$ the highest root of $R_{S}$.

Let $S \subset \Pi$. We define inductively a set $\mathcal{K}(S)$ whose elements are subsets of $\Pi$ as follows:

a) $\mathcal{K}(\emptyset)=\emptyset$

b) If $S_{1}, \ldots, S_{r}$ are the connected components of $S$, then $\mathcal{K}(S)=\mathcal{K}\left(S_{1}\right) \cup \cdots \cup$ $\mathcal{K}\left(S_{r}\right)$.

c) If $S$ is connected, then $\mathcal{K}(S)=\{S\} \cup \mathcal{K}(\widehat{S})$ where $\widehat{S}=\left\{\alpha \in S ;\left\langle\alpha, \varepsilon_{S}^{\vee}\right\rangle=0\right\}$.

It is well known that (see for example [14, Chapter 40]) elements of $\mathcal{K}(S)$ are connected subsets of $S$. Moreover, if we denote by $\mathcal{R}(S)=\left\{\varepsilon_{K} ; K \in \mathcal{K}(S)\right\}$, then $\mathcal{R}(S)$ is a maximal set of pairwise strongly orthogonal roots in $R_{S}$.

Let us also recall the following properties of $\mathcal{K}(S)$ which are easy consequences from the definition (see for example [14, Chapter 40]):

Lemma 1.1. Let $S$ be a subset of $\Pi, K, K^{\prime} \in \mathcal{K}(S)$ and set

$$
\begin{aligned}
\Gamma^{K} & =\left\{\alpha \in R_{K} ;\left\langle\alpha, \varepsilon_{K}^{\vee}\right\rangle>0\right\} \\
& =\left\{\alpha=\sum_{\beta \in K} n_{\beta} \beta \in R_{K}^{+} ; n_{\beta}>0 \text { for some } \beta \in K \backslash \widehat{K}\right\} .
\end{aligned}
$$

i) We have either $K \subset K^{\prime}$ or $K^{\prime} \subset K$ or $K$ and $K^{\prime}$ are connected components of $K \cup K^{\prime}$.

ii) $\Gamma^{K}=R_{K}^{+} \backslash\left\{\beta \in R_{K}^{+} ;\left\langle\beta, \varepsilon_{K}^{\vee}\right\rangle=0\right\}$. In particular, $R_{S}^{+}$is the disjoint union of the $\Gamma^{K}$ 's, $K \in \mathcal{K}(S)$. 
TABLE 1

\begin{tabular}{|c|c|c|c|c|c|c|c|c|}
\hline Type & $A_{\ell}, \ell \geq 1$ & $B_{\ell}, C_{\ell}, \ell \geq 2$ & $D_{\ell}, \ell \geq 4$ & $E_{6}$ & $E_{7}$ & $E_{8}$ & $F_{4}$ & $G_{2}$ \\
\hline$\sharp \mathcal{K}(\Pi)$ & {$\left[\frac{\ell+1}{2}\right]$} & $\ell$ & $2\left[\frac{\ell}{2}\right]$ & 4 & 7 & 8 & 4 & 2 \\
\hline
\end{tabular}

iii) $\sum_{\alpha \in \Gamma^{K}} \mathfrak{g}_{\alpha}$ is a Heisenberg Lie algebra whose centre is $\mathfrak{g}_{\varepsilon_{K}}$. Thus if $\alpha, \beta \in \Gamma^{K}$ verify $\alpha+\beta \in R$, then $\alpha+\beta=\varepsilon_{K}$.

iv) Suppose that $\alpha \in \Gamma^{K}$ and $\beta \in \Gamma^{K^{\prime}}$ verify $\alpha+\beta \in R$, then either $K \subset K^{\prime}$ and $\alpha+\beta \in \Gamma^{K^{\prime}}$ or $K^{\prime} \subset K$ and $\alpha+\beta \in \Gamma^{K}$.

The cardinality of $\mathcal{K}(\Pi)$ is listed in Table 1 for an irreducible root system $R$, where for any $x \in \mathbb{Q},[x]$ is a unique integer such that $[x] \leq x<[x]+1$.

Examples 1.2. Let $R$ be an irreducible root system. We shall use the numbering of simple roots in [14, Chapter 18]. Set $k=\sharp \mathcal{K}(\Pi)$.

$1)$ Let $R$ be of type $A_{\ell}$. Then $\mathcal{K}(\Pi)=\bigcup_{i=1}^{k}\left\{K_{i}\right\}$ where $K_{i}=\left\{\alpha_{i}, \ldots, \alpha_{\ell+1-i}\right\}$. For $1 \leq i \leq k$,

$$
\Gamma^{K_{i}}=\left\{\alpha_{i}+\cdots+\alpha_{i+r}, \alpha_{\ell+1-i-r}+\cdots+\alpha_{\ell+1-i} ; 0 \leq r \leq \ell-2 i\right\} \cup\left\{\varepsilon_{K_{i}}\right\} .
$$

2) Let $R$ be of type $D_{2 n+1}$. Then $k=2 n$,

$$
\mathcal{K}(\Pi)=\bigcup_{i=1}^{n}\left\{K_{i}\right\} \cup\left\{L_{i}\right\},
$$

where $K_{i}=\left\{\alpha_{2 i-1}, \ldots, \alpha_{2 n+1}\right\}$ and $L_{i}=\left\{\alpha_{2 i-1}\right\}$. For $1 \leq i \leq n$,

$$
\Gamma^{K_{i}}=\left\{\sum_{j=2 i-1}^{\ell} m_{j} \alpha_{j} ; m_{2 i} \neq 0\right\}, \quad \Gamma^{L_{i}}=\left\{\alpha_{2 i-1}\right\} .
$$

3) Let $R$ be of type $E_{6}$. Then

$$
\mathcal{K}(\Pi)=\{\Pi\} \cup\left\{\left\{\alpha_{1}, \alpha_{3}, \alpha_{4}, \alpha_{5}, \alpha_{6}\right\}\right\} \cup\left\{\left\{\alpha_{3}, \alpha_{4}, \alpha_{5}\right\}\right\} \cup\left\{\left\{\alpha_{4}\right\}\right\} .
$$

\section{MAIN RESUlT}

Recall that for any subset $S \subset \Pi$,

$$
\mathfrak{p}_{S}=\mathfrak{h} \oplus \bigoplus_{\alpha \in R_{S} \cup R^{+}} \mathfrak{g}_{\alpha}
$$

is a (standard) parabolic subalgebra of $\mathfrak{g}$. Any parabolic subalgebra of $\mathfrak{g}$ is conjugated to a standard parabolic subalgebra. The Lie subalgebra

$$
\mathfrak{u}_{S}=\bigoplus_{\alpha \in R^{+} \backslash R_{S}} \mathfrak{g}_{\alpha}
$$

is the nilpotent radical of $\mathfrak{p}_{S}$.

For $S \subset \Pi$, denote by $V_{S}$ the vector subspace of $\mathfrak{h}^{*}$ spanned by the elements of $\mathcal{R}(S)$ and $\mathcal{R}(\Pi)$. Set

$$
\begin{gathered}
\mathcal{E}_{S}=\left\{K \in \mathcal{K}(\Pi) ; X_{\varepsilon_{K}} \in \mathfrak{u}_{S}\right\}=\left\{K \in \mathcal{K}(\Pi) ; \varepsilon_{K} \notin R_{S}\right\} \\
\text { and } \mathcal{Q}_{S}=\left(\bigcup_{K \in \mathcal{E}_{S}} \Gamma^{K}\right) \cap R_{S}^{+} .
\end{gathered}
$$

The subset $\mathcal{E}_{S}$ has the following simple characterisation. 
Lemma 2.1. Let $T_{S}$ be the union of the subsets $K \in \mathcal{K}(\Pi)$ verifying $K \subset S$. Then $\mathcal{E}_{S}=\mathcal{K}(\Pi) \backslash \mathcal{K}\left(T_{S}\right)$.

Proof. This is straightforward.

Our main result is the following theorem.

Theorem 2.2. Let $S \subset \Pi$. Then

$$
\chi\left(\mathfrak{p}_{S}\right)+\chi\left(\mathfrak{u}_{S}\right)=\operatorname{rk} \mathfrak{g}+\sharp \mathcal{K}(S)-\sharp \mathcal{K}\left(T_{S}\right)+2\left(\sharp \mathcal{K}(\Pi)-\operatorname{dim} V_{S}\right)+\sharp \mathcal{Q}_{S} .
$$

We have $\chi\left(\mathfrak{p}_{S}\right)+\chi\left(\mathfrak{u}_{S}\right) \geq \mathrm{rk} \mathfrak{g}$, and the equality holds if and only if the following conditions are satisfied:

i) $\sharp(\mathcal{K}(S) \cup \mathcal{K}(\Pi))=\operatorname{dim} V_{S}$.

ii) For any connected component $S^{\prime}$ of $S$, we have either $S^{\prime} \in \mathcal{K}(\Pi)$ or $\sharp\left(S^{\prime} \backslash T_{S}\right)=$ 1.

Proof. We may clearly assume that $\mathfrak{g}$ is simple.

The formula for the sum $\chi\left(\mathfrak{p}_{S}\right)+\chi\left(\mathfrak{u}_{S}\right)$ is a direct consequence of the formula of the index of parabolic subalgebras conjectured in [13] and proved in $[3,6]$ :

$$
\chi\left(\mathfrak{p}_{S}\right)=\operatorname{rk} \mathfrak{g}+\sharp \mathcal{K}(\Pi)+\sharp \mathcal{K}(S)-2 \operatorname{dim} V_{S},
$$

and the formula for the index of $\mathfrak{u}_{S}$ (see for example [14, Chapter 40]), which, in view of Lemma 1.1, can be expressed in the following way:

$$
\chi\left(\mathfrak{u}_{S}\right)=\sharp \mathcal{E}_{S}+\sum_{K \in \mathcal{E}_{S}} \sharp \Gamma^{K}-\operatorname{dim} \mathfrak{u}_{S}=\sharp \mathcal{E}_{S}+\sharp \mathcal{Q}_{S} .
$$

Observe that

$$
\sharp \mathcal{K}(S)-\sharp \mathcal{K}\left(T_{S}\right) \geq 0 .
$$

1) Let $S_{1}, \ldots, S_{r}$ be the connected components of $S$. For each $i$, there is a unique $K_{i} \in \mathcal{K}(\Pi)$ (see Lemma 1.1) such that $\varepsilon_{S_{i}} \in \Gamma^{K_{i}}$. If $K_{i}=S_{i}$, then $S_{i}$ is a connected component of $T_{S}$. Otherwise $K_{i} \in \mathcal{E}_{S}$, and we have

$$
\varepsilon_{S_{i}} \in \Gamma^{K_{i}} \cap \Gamma^{S_{i}} \subset \mathcal{Q}_{S} .
$$

2) It follows from Point 1) that $\mathcal{Q}_{S}=\emptyset$ if and only if $\mathcal{K}(S) \subset \mathcal{K}(\Pi)$ (or equivalently $S=T_{S}$ ).

3) Note that the connected components of $T_{S}$ are the connected components of $T_{S} \cap S_{i}$. It follows again from Point 1) that

$$
\begin{aligned}
\sharp \mathcal{K}(S)-\sharp \mathcal{K}\left(T_{S}\right) & =\sum_{i=1}^{r}\left(\sharp \mathcal{K}\left(S_{i}\right)-\sharp \mathcal{K}\left(T_{S} \cap S_{i}\right)\right) \\
& =\sum_{K_{i} \in \mathcal{E}_{S}}\left(\sharp \mathcal{K}\left(S_{i}\right)-\sharp \mathcal{K}\left(T_{S} \cap S_{i}\right)\right) .
\end{aligned}
$$

4) From Table 1, we have $\operatorname{dim} V_{S}=\sharp \mathcal{K}(\Pi)=\operatorname{rk} \mathfrak{g}$ in the cases where $\mathfrak{g}$ is of type $B_{\ell}, C_{\ell}, D_{2 n}, E_{7}, E_{8}, F_{4}$ and $G_{2}$. The inequality follows immediately from (3) and (6), and by Point 2) the equality holds if and only if $\mathcal{K}(S) \subset \mathcal{K}(\Pi)$.

On the other hand, since $\operatorname{dim} V_{S}=\sharp \mathcal{K}(\Pi)$ in these cases, condition i) is equivalent to $\mathcal{K}(S) \subset \mathcal{K}(\Pi)$. Finally, if condition i) is verified, then $S=T_{S}$, and condition ii) is automatically verified. So we have the result in these cases.

5) Type $A_{\ell}$. 
For any $i$ verifying $S_{i} \neq K_{i}$, by Point 1), Lemma 1.1 and Examples 1.2, half of $\Gamma^{S_{i}} \backslash\left\{\varepsilon_{S_{i}}\right\}$ belongs to $\mathcal{Q}_{S}$. Since $\sharp\left(\Gamma^{S_{i}}\right)=2 \sharp\left(S_{i}\right)-1$ (Examples 1.2), such an $S_{i}$ contributes $\sharp\left(S_{i}\right)$ elements of $\mathcal{Q}_{S}$.

Again, since we are in type $A_{\ell}, \mathcal{K}(\Pi)$ is totally ordered by inclusion. It follows that $T_{S}$ is connected. Without loss of generality, we may assume that $T_{S} \subset S_{1}$.

Suppose that $S_{1}=T_{S}$. Then from the previous discussion, we deduce that

$$
\chi\left(\mathfrak{p}_{S}\right)+\chi\left(\mathfrak{u}_{S}\right) \geq \operatorname{rkg}+\sharp \mathcal{K}\left(S \backslash S_{1}\right)+2\left(\sharp \mathcal{K}(\Pi)-\operatorname{dim} V_{S}\right)+\sharp\left(S \backslash S_{1}\right) .
$$

But our hypothesis implies that

$$
\operatorname{dim} V_{S} \leq \sharp \mathcal{K}(\Pi)+\sharp \mathcal{K}\left(S \backslash S_{1}\right)=\sharp(\mathcal{K}(\Pi) \cup \mathcal{K}(S)),
$$

so

$$
\chi\left(\mathfrak{p}_{S}\right)+\chi\left(\mathfrak{u}_{S}\right) \geq \operatorname{rkg}+\sharp\left(S \backslash S_{1}\right)-\sharp \mathcal{K}\left(S \backslash S_{1}\right) .
$$

Hence $\chi\left(\mathfrak{p}_{S}\right)+\chi\left(\mathfrak{u}_{S}\right) \geq \operatorname{rk} \mathfrak{g}$. For the equality to hold, we must have the equality in (7) and

$$
\sharp\left(S \backslash S_{1}\right)=\sharp \mathcal{K}\left(S \backslash S_{1}\right) .
$$

This latter is only possible if $\sharp\left(S_{i}\right)=1$ for $i \geq 2$, so we have conditions (i) and (ii). Conversely, suppose that conditions (i) and (ii) are verified; then $\sharp\left(S_{i}\right)=1$ for $i \geq 2$. Consequently $\mathcal{Q}_{S}=S \backslash S_{i}$ by Point 1 ) and the definition of $\mathcal{Q}_{S}$.

Suppose that $S_{1} \supsetneq T_{S}$ (this includes the case $T_{S}=\emptyset$ ). Then $\mathcal{K}(S) \cap \mathcal{K}(\Pi)=\emptyset$. Thus

$$
\operatorname{dim} V_{S} \leq \sharp \mathcal{K}(\Pi)+\sharp \mathcal{K}(S)=\sharp(\mathcal{K}(\Pi) \cup \mathcal{K}(S)) .
$$

We deduce from Point 1) and the remark in the first paragraph of Point 5) that

$$
\begin{aligned}
\chi\left(\mathfrak{p}_{S}\right)+\chi\left(\mathfrak{u}_{S}\right) & \geq \operatorname{rkg}+\sharp \mathcal{K}(S)-\sharp \mathcal{K}\left(T_{S}\right)+2\left(\sharp \mathcal{K}(\Pi)-\operatorname{dim} V_{S}\right)+\sharp(S) \\
& \geq \operatorname{rkg}+\sharp(S)-\sharp \mathcal{K}(S)-\sharp \mathcal{K}\left(T_{S}\right) .
\end{aligned}
$$

Hence

$$
\chi\left(\mathfrak{p}_{S}\right)+\chi\left(\mathfrak{u}_{S}\right) \geq \operatorname{rkg}+\sum_{i=1}^{r}\left(\sharp\left(S_{i}\right)-\left[\frac{\sharp\left(S_{i}\right)+1}{2}\right]\right)-\left[\frac{\sharp\left(T_{S}\right)+1}{2}\right] .
$$

Since $T_{S} \subsetneq S_{1}$, we deduce from Table 1 that

$$
\sharp\left(S_{1}\right)-\left[\frac{\sharp\left(S_{1}\right)+1}{2}\right]-\left[\frac{\sharp\left(T_{S}\right)+1}{2}\right] \geq 0 .
$$

So we have our inequality $\chi\left(\mathfrak{p}_{S}\right)+\chi\left(\mathfrak{u}_{S}\right) \geq \mathrm{rk} \mathfrak{g}$.

Now for the equality $\chi\left(\mathfrak{p}_{S}\right)+\chi\left(\mathfrak{u}_{S}\right)=$ rk $\mathfrak{g}$ to hold, we must have the equality in (8) and $\sharp \mathcal{Q}_{S}=\sharp(S)$,

$$
\sharp\left(S_{1}\right)-\left[\frac{\sharp\left(S_{1}\right)+1}{2}\right]-\left[\frac{\sharp\left(T_{S}\right)+1}{2}\right]=0 \text { and } \sharp\left(S_{i}\right)-\left[\frac{\sharp\left(S_{i}\right)+1}{2}\right]=0
$$

for $i \geq 2$. This implies that $\sharp\left(S_{1}\right)=\sharp\left(T_{S}\right)+1$, and $\sharp\left(S_{i}\right)=1$ for $i \geq 2$. So we have conditions (i) and (ii). Conversely, if conditions (i) and (ii) are verified, then $\sharp\left(S_{i}\right)=1$ for $i \geq 2$, and $\sharp\left(S_{1}\right)=\sharp\left(T_{S}\right)+1$. In view of the above, to show that $\chi\left(\mathfrak{p}_{S}\right)+\chi\left(\mathfrak{u}_{S}\right)=\mathrm{rk} \mathfrak{g}$, it suffices to check that $\sharp\left(\mathcal{Q}_{S} \cap R_{S_{1}}^{+}\right)=\sharp\left(S_{1}\right)$, which is a straightforward verification.

6) Type $D_{2 n+1}$.

In this case, $\sharp \mathcal{K}(\Pi)=\mathrm{rk} \mathfrak{g}-1$. Let us use the numbering of simple roots in $[14$, Chapter 18]. We check easily that $\alpha_{1}, \ldots, \alpha_{\ell-2} \in V_{\Pi}$. 
TABLE 2

\begin{tabular}{|c|c|c|c|c|c|c|}
\hline$S_{1}$ & $\operatorname{dim} V_{S_{1}}$ & $T_{S} \cap S_{1}$ & $\sharp \mathcal{K}\left(S_{1}\right)$ & $\sharp \mathcal{K}\left(T_{S} \cap S_{1}\right)$ & $K_{1}$ & $\sharp\left(\Gamma^{K_{1}} \cap \Gamma^{S_{1}}\right)$ \\
\hline$A_{1}$ & 5 & $\emptyset$ & 1 & 0 & $A_{3}$ or $A_{5}$ & 1 \\
\hline$A_{2}$ & 5 & $\emptyset$ & 1 & 0 & $A_{5}$ & 2 \\
\hline$A_{2}$ & 5 & $A_{1}$ & 1 & 1 & $A_{3}$ & 2 \\
\hline$A_{3}$ & 6 & $A_{1}$ & 2 & 1 & $A_{5}$ & 3 \\
\hline$A_{3}$ & 5 & $A_{1}$ & 2 & 1 & $E_{6}$ & 3 \\
\hline$A_{4}$ & 6 & $A_{1}$ & 2 & 1 & $E_{6}$ & 4 \\
\hline$A_{4}$ & 6 & $A_{3}$ & 2 & 2 & $A_{5}$ & 4 \\
\hline$D_{4}$ & 5 & $A_{3}$ & 4 & 2 & $E_{6}$ & 4 \\
\hline$D_{5}$ & 6 & $A_{3}$ & 4 & 2 & $E_{6}$ & 9 \\
\hline
\end{tabular}

If $\operatorname{dim} V_{S}=\sharp \mathcal{K}(\Pi)$, then the inequality follows from (3) and (6), and the condition for equality follows from Point 2).

Suppose now that $\operatorname{dim} V_{S}=\operatorname{rkg}$ and $\alpha_{\ell-1} \in S$ (the case $\alpha_{\ell} \in S$ being analogous). Then

$$
\chi\left(\mathfrak{p}_{S}\right)+\chi\left(\mathfrak{u}_{S}\right)=\operatorname{rk} \mathfrak{g}+\sharp \mathcal{K}(S)-\sharp \mathcal{K}\left(T_{S}\right)-2+\sharp\left(\mathcal{Q}_{S}\right),
$$

and the connected component $S_{1}$ of $S$ containing $\alpha_{\ell-1}$ is not in $\mathcal{K}(\Pi)$; otherwise, we would have $\operatorname{dim} V_{S}=\sharp \mathcal{K}(\Pi)$.

By Point 1), $\varepsilon_{S_{1}} \in \mathcal{Q}_{S}$. By examining the possibilities for $S_{1}$ and $K_{1}$ (Examples $1.2)$, we verify that

$$
\sharp \mathcal{K}\left(S_{1}\right)-\sharp \mathcal{K}\left(T_{S} \cap S_{1}\right)+\sharp\left(\Gamma^{K_{1}} \cap \Gamma^{S_{1}}\right) \geq 2
$$

with equality if and only if $S_{1}$ is of type $A_{1}$ or $A_{2}$. Therefore, we have obtained the inequality.

In fact, we showed in the previous paragraph that already we have

$$
\operatorname{rkg}+\sharp \mathcal{K}\left(S_{1}\right)-\sharp \mathcal{K}\left(T_{S} \cap S_{1}\right)-2+\sharp\left(\Gamma^{K_{1}} \cap \Gamma^{S_{1}}\right) \geq \operatorname{rk} \mathfrak{g} .
$$

So if $\chi\left(\mathfrak{p}_{S}\right)+\chi\left(\mathfrak{u}_{S}\right)=\mathrm{rk} \mathfrak{g}$, then from (9) and the above inequality, we must have $\mathcal{K}\left(S \backslash S_{1}\right) \subset \mathcal{K}(\Pi)$, and also the equality in (10). Hence conditions (i) and (ii). Conversely, suppose that conditions (i) and (ii) are verified; then the fact that $\alpha_{1}, \ldots, \alpha_{\ell-2} \in V_{\Pi}$ implies that $\mathcal{K}\left(S \backslash S_{1}\right) \subset \mathcal{K}(\Pi)$ and $\sharp \mathcal{K}\left(S_{1}\right)=1$. Hence $S_{1}$ is of type $A_{1}, A_{2}$. It is then easy to check that $\chi\left(\mathfrak{p}_{S}\right)+\chi\left(\mathfrak{u}_{S}\right)=$ rk $\mathfrak{g}$.

7) Type $E_{6}$.

Here, we have $\sharp \mathcal{K}(\Pi)=4$ and $\alpha_{2}, \alpha_{4} \in V_{\Pi}$. Let $S_{1}$ be a connected component of $S$ such that $\operatorname{dim} V_{S_{1}}>4$. Under these conditions, the possibilities are shown in Table 2. Thus,

$$
\sharp \mathcal{K}\left(S_{1}\right)-\sharp \mathcal{K}\left(T_{S} \cap S_{1}\right)+\sharp\left(\Gamma^{S_{1}} \cap \Gamma^{K_{1}}\right) \geq 2\left(\operatorname{dim} V_{S}-\sharp \mathcal{K}(\Pi)\right) .
$$

A direct verification gives the result. Note that as in the case of type $A_{\ell}, \mathcal{K}(\Pi)$ is totally ordered by inclusion, so $T_{S}$ is connected.

Remark 2.3. Theorem 2.2 says that if $\mathcal{K}(S) \subset \mathcal{K}(\Pi)$ or, equivalently, $S^{\prime} \in \mathcal{K}(\Pi)$ for any connected component $S^{\prime}$ of $S$, then $\chi\left(\mathfrak{p}_{S}\right)+\chi\left(\mathfrak{u}_{S}\right)=$ rk $\mathfrak{g}$. 
TABLE 3

\begin{tabular}{|c|c|c|}
\hline Type & $\mathcal{K}(S) \not \subset \mathcal{K}(\Pi)$ & $\mathcal{K}(S) \subset \mathcal{K}(\Pi)$ \\
\hline$A_{\ell}$ & $i \neq \frac{\ell+1}{2}$ & $i=\frac{\ell+1}{2}$ \\
\hline$B_{\ell}$ & none & $i$ odd \\
\hline$C_{\ell}$ & none & $i=\ell$ \\
\hline$D_{2 n+1}$ & $i=2 n, 2 n+1$ & $i<2 n$ odd \\
\hline$D_{2 n}$ & none & $i$ odd or $i=2 n$ \\
\hline$E_{6}$ & $i \neq 2,4$ & $i=4$ \\
\hline$E_{7}$ & none & $i=2,3,5,7$ \\
\hline$E_{8}$ & none & $i=2,3,5,7$ \\
\hline$F_{4}$ & none & $i=2$ \\
\hline$G_{2}$ & none & $i=1$ \\
\hline
\end{tabular}

Example 2.4. Let us consider the case of minimal parabolic subalgebras. So $S=\{\alpha\}$ and it follows that

$$
\chi(\mathfrak{p})+\chi(\mathfrak{u})= \begin{cases}\operatorname{rkg} & \text { if }\{\alpha\} \in \mathcal{K}(\Pi), \\ \operatorname{rkg} & \text { if }\{\alpha\} \notin \mathcal{K}(\Pi) \text { and } \operatorname{dim} V_{S}=\sharp \mathcal{K}(\Pi)+1, \\ \operatorname{rkg}+2 & \text { if }\{\alpha\} \notin \mathcal{K}(\Pi) \text { and } \operatorname{dim} V_{S}=\sharp \mathcal{K}(\Pi) .\end{cases}
$$

Thus the minimal parabolic subalgebras $\mathfrak{p}_{S}$ verifying $\chi\left(\mathfrak{p}_{S}\right)+\chi\left(\mathfrak{u}_{S}\right)=\operatorname{rk} \mathfrak{g}$ are (in the simple roots numbering of [14, Chapter 18]) as shown in Table 3.

Example 2.5. In the other extreme, it is easy to check that maximal parabolic subalgebras of $\mathfrak{g}=\operatorname{sl}_{\ell+1}$ verifying $\chi(\mathfrak{p})+\chi(\mathfrak{u})=$ rk $\mathfrak{g}$ are exactly the ones associated to simple roots at the extremities of the Dynkin diagram.

\section{ACKNOWLEDGMENT}

The author would like to thank the anonymous referee for his or her remarks.

\section{REFERENCES}

[1] Charbonnel J.-Y., Propriétés (Q) et (C). Variété commutante, Bull. Soc. Math. France, 132 (2004) 477-508. MR2131901 (2006b:14079)

[2] Dergachev V. and Kirillov A., Index of Lie algebras of seaweed type, J. of Lie Theory, 10 (2000) 331-343. MR1774864 (2001j:17014)

[3] Fauquant-Millet F. And Joseph A., La somme des faux degrés - un mystère en théorie des invariants, preprint (2005).

[4] Jantzen J.C., Einhüllenden Algebren halbeinfacher Lie-Algebren, in : Ergebnisse der Mathematik and iher Grenzgebiete, 3, Springer Verlag, 1983. MR721170 (86c:17011)

[5] Joseph A., A preparation theorem for the prime spectrum of a semisimple Lie algebra, J. of Algebra, 48 (1977) 241-289. MR0453829 (56:12082)

[6] Joseph A., On semi-invariants and index for biparabolic (seaweed) algebras I, J. of Algebra, 305 (2006) 487-515. MR2264140 (2007f:17033)

[7] Moreau A., Indice du normalisateur du centralisateur d'un élément nilpotent dans une algèbre de Lie semi-simple, Bull. Soc. Math. France, 134 (2006) 83-117. MR2233701 (2007e:17006)

[8] Moreau A., Indice et décomposition de Cartan d'une algèbre de Lie semisimple réelle, J. of Algebra, 303 (2006) 382-406. MR2253668 (2007d:17007)

[9] Panyushev D., Inductive formulas for the index of seaweed Lie algebras, Moscow Math. Journal, 1 (2001) 221-241. MR1878277 (2002k:17020) 
[10] Panyushev D., The index of a Lie algebra, the centralizer of a nilpotent element, and the normalizer of the centralizer, Math. Proc. Camb. Phil. Soc., 134 (2003) 41-59. MR1937791 (2003i:17006)

[11] RAÏs M., Notes sur l'indice des algèbres de Lie (I) et (II), preprints arXiv math.RT/0605499 and math.RT/0605500.

[12] Tauvel P. And Yu R.W.T., Indice et formes linéaires stables dans les algèbres de Lie, J. of Algebra, 273 (2004) 507-516. MR2037708 (2005h:17010)

[13] Tauvel P. and Yu R.W.T., Sur l'indice de certaines algèbres de Lie, Annales de l'Institut Fourier, 54 (2004) 1793-1810. MR2134224 (2005m:17003)

[14] TAuvel P. AND Yu R.W.T., Lie algebras and algebraic groups, Springer Monographs in Mathematics, Springer Verlag, Berlin, 2005. MR2146652 (2006c:17001)

[15] Yakimova O., The index of centralisers of elements in classical Lie algebras, Funct. Analysis and its Applications, 40 (2006) 42-51. MR2223249 (2007d:17008)

UMR 6086 Du C.N.R.S., Département de Mathématiques, Université de Poitiers, Téléport 2 - BP 30179, Boulevard Marie et Pierre Curie, 86962 Futuroscope ChasSeneuil Cedex, France

E-mail address: yuyu@math.univ-poitiers.fr 